\title{
School Age Child
}

National Cancer Institute

\section{Source}

National Cancer Institute. School Age Child. NCI Thesaurus. Code C85405.

A child between the ages of five and twelve years. 